Research article

\title{
Task difficulty has no effect on haptic anchoring during tandem walking in young and older adults
}

\author{
Andréia Abud da Silva Costa ${ }^{\mathrm{a}, \mathrm{b}}$, Luciana Oliveira dos Santos ${ }^{\mathrm{a}, \mathrm{b}}$, Eliane Mauerberg-deCastro ${ }^{\mathrm{c}}$, \\ Renato Moraes ${ }^{\mathrm{b} \text {, }}$ \\ ${ }^{a}$ Ribeirão Preto Medical School, Graduate Program in Rehabilitation and Functional Performance, University of São Paulo, Brazil \\ b Biomechanics and Motor Control Lab, School of Physical Education and Sport of Ribeirão Preto, University of São Paulo, Brazil \\ ${ }^{\mathrm{c}}$ Department of Physical Education, Biosciences Institute, São Paulo State University, Brazil
}

\section{A R T I C L E I N F O}

\section{Keywords:}

Older adults

Anchor system

Haptic information

Trunk acceleration

Tandem gait

Balance beam

\begin{abstract}
A B S T R A C T
This study assessed the contribution of the "anchor system's" haptic information to balance control during walking at two levels of difficulty. Seventeen young adults and seventeen older adults performed 20 randomized trials of tandem walking in a straight line, on level ground and on a slightly-raised balance beam, both with and without the use of the anchors. The anchor consists of two flexible cables, whose ends participants hold in each hand, to which weights $(125 \mathrm{~g})$ are attached at the opposing ends, and which rest on the ground. As the participants walk, they pull on the cables, dragging the anchors. Spatiotemporal gait variables (step speed and single- and double-support duration) were processed using retro-reflective markers on anatomical sites. An accelerometer positioned in the cervical region registered trunk acceleration. Walking on the balance beam increased single- and double-support duration and reduced step speed in older adults, which suggests that this condition was more difficult than walking on the level ground. The anchors reduced trunk acceleration in the frontal plane, but the level of difficulty of the walking task showed no effect. Thus, varying the difficulty of the task had no influence on the way in which participants used the anchor system while tandem walking. The older adults exhibited more difficulty in walking on the balance beam as compared to the younger adults; however, the effect of the anchor system was similar in both groups.
\end{abstract}

\section{Introduction}

When compared to young adults, older adults show an increase in frontal plane sway during walking due to difficulties in medial-lateral balance control [1], which increases the risk for falls [2]. Previous studies that analyzed gait patterns found that trunk variability increases with age $[3,4]$ and discriminates between healthy and frail older adults [5]. Recently, we investigated how additional haptic information that an "anchor system" provides [6] affects older adults' trunk sway during tandem walking on the ground in a straight line (i.e., placing one foot in front of the other) [7]. Acceleration in the frontal plane was reduced when participants used the anchors.

The anchor system, a non-rigid tool designed to help reduce body sway, has been studied in different groups, including older adults $[7,8,9]$, with the purpose of testing the extent of the integration of haptic cues by the postural control system, whether in static balance tasks or dynamic locomotion tasks. The anchor consists of a flexible cable held in each hand, with an endpoint attached to a light mass, which is kept in contact with a surface (typically, the floor or ground) [6]. During proper use of the anchors, the cable should be kept taut and the mass should touch the ground at all times. In balancing tasks, the dynamic interplay between the cables' varying tensions and the continuous hand-pulling adjustments and body oscillations is the source of the haptic input that the postural system integrates in order to reduce body sway [10]. The anchors provide information about spatial orientation of the body relative to the ground, which helps to maintain the trunk in the upright position [7,10]. Information, in this case, is understood as a pattern of a continuous flow of energy that specifies the environment's constraints to the individual [10].

The effect of the anchors has been studied in quiet standing tasks in young and older adults $[6,8,9]$. However, the anchors have the potential to be used in walking tasks $[7,11,12]$. This is important, since the most falls by older adults occur during walking [13]. Studies involving participants that lightly touched rigid and non-rigid surfaces while walking showed that additional haptic cues improve balance control $[14,15,16]$. Such tasks require the continuous touch of an individual's

\footnotetext{
* Corresponding author at: Escola de Educação Física e Esporte de Ribeirão Preto, Universidade de São Paulo, Av. Bandeirantes, 3900, Ribeirão Preto, SP, Brazil

E-mail address: renatomoraes@usp.br (R. Moraes).
} 
fingers on a surface for haptic input to occur. Recently, Hedayat et. al [12] showed that, during walking by young adults, the effects of the anchors were more beneficial in improving balance control in the frontal plane than were those from when they lightly touched a railing. Also, an important observation from this study was that in both the condition with hands holding the anchors and the condition with hands positioned similarly but not holding the anchors, trunk velocity in the frontal plane was reduced. However, the use of the anchors showed a more pronounced reduction in trunk velocity. This result suggests that it is not only the mechanical consequences of the task's arm/hand configuration that contributed to reduce frontal plane trunk velocity, but that the haptic cues from the anchors make a distinct contribution to the postural control system. Similarly, using a placebo anchor (i.e., holding the cable without any load on the other extremity) did not reduce trunk acceleration amplitude in the frontal plane in older adults [7].

The anchors can be easily used in different contexts, and, while in this case haptic input requires a reference surface (i.e., the ground), the various degrees of freedom for each arm allow control strategies to operate under flexible task contexts. That is, even in the event of high amplitudes of body sway, haptic anchoring is a coupled system (a mass attached to a cable, which is attached to the hand/fingers, linked biomechanically to adjacent segments of the arm and torso) that allows individuals to dynamically adjust their control strategies without abandoning the task (i.e., releasing the cables). While lightly touching a surface during a walking task, continuous direct contact between the fingers and the surface requires precision. Therefore, to some extent, even small disruptions caused by body sway can interrupt the task (i.e., releasing the connection between fingers and surface), preventing haptic input.

Although we recently showed that older adults could benefit from the anchors to improve balance control during walking, we failed to show any transfer to a post-practice condition without the anchors [7]. In that study, we suggested that this limitation in transfer effect could be the result of the walking task's low level of difficulty. Benefits of the anchors are less evident during the practice of simple postural tasks (e.g., standing with feet apart) as compared to tasks that challenged balance control (e.g., standing with one foot, with feet together, or with eyes closed) [10,17]. Our previous study [7] showed that tandem walking in a straight line on level ground was not challenging enough for the participants to exploit the additional haptic input provided by the anchors, although this task caused destabilization of their posture. By investigating varied levels of task difficulty and how individuals exploit haptic information provided via the anchors, we could assess the extent of optimal task demands and their respective behavioral outcomes. The amount and types of challenges in walking tasks are factors to consider when designing an intervention protocol [18]. In locomotion tasks, increasing the difficulty level can be tested by simply asking participants to walk on a balance beam.

Different studies employing tandem walking tasks showed that reduced width of the base of support result in increased variability of trunk displacement $[1,19]$. When young and older adults walked on a treadmill with their step width reduced by $50 \%$ from its normal width, their instability increased, as did their variability of body oscillation [3]. Tandem walking and walking on a balance beam are similar only in the requirement for specific placement of the foot along a straight line. Individuals who perform a tandem walking task on a balance beam have the additional challenge of accurately placing the feet on the beam while maintaining balance $[18,20]$. Also, there likely is a detrimental influence of the emotional factor of knowing that they are walking on a surface of a certain height [21]. Many older individuals are afraid of small changes in height, as they perceive them to be risks for falls. Tandem walking on a balance beam seems to be a relatively simple task; yet, for some individuals (i.e., disabled or older individuals) it is difficult enough to challenge the balance control mechanism [20]. Walking on a balance beam prompts lateral instability [18], increases trunk sway, and is a condition that tests whether the additional haptic input that the anchors provide is effective in improving walking performance.

Our purpose was to assess balance control in young and older adults in tandem walking tasks with two levels of difficulty (ground and balance beam) while exploiting additional haptic information provided by the anchors. We expected that the anchors would reduce trunk sway acceleration in the frontal plane and double-support phase duration in both young and older adults, especially on the balance beam. We also expected that the older adults, due to the challenge of the locomotion tasks, would better exploit the anchor system than would the young adults, particularly while tandem walking on the balance beam. Studying the extent to which the anchors could provide benefits during walking by these individuals could potentially enrich rehabilitation techniques designed to improve dynamic balance in intervention programs.

\section{Material and methods}

\subsection{Participants}

Seventeen young adults (18-29 years old, 7 females and 10 males) and 17 older adults (65-77 years old, 11 females and 6 males) participated in this study. The procedures of this study were approved by the Ethics Committee. Participants had to be able to understand the verbal instructions to accomplish the tasks and to walk independently. Exclusion criteria were: cognitive impairment, stroke, cardiovascular diseases, orthopedic injuries, limited trunk mobility, severe vision loss or glaucoma, and impairments of the vestibular or somatosensory system. These criteria were assessed via a questionnaire applied before the data collection. To assess the level of physical activity, we applied the Baecke Questionnaire [22] for younger adults and the modified Baecke Questionnaire for older adults [23]. These questionnaires assess physical activities related to housework and work activities, sport and leisure activities. We also applied the Mini Mental State Examination [24] to assess cognitive function and the MiniBEST Test [25] to assess the functional balance of the older adults. Hand and foot tactile sensitivity of the older adults was measured with an esthesiometer (SORRI, Bauru, Brazil) [26].

\subsection{Procedures}

Six reflective markers were placed bilaterally on the 5th metatarsal, and the lateral malleolus and calcaneus (Fig. 1A). Each marker's displacement was recorded by a motion capture system composed of eight infrared cameras (MX-T40S, Vicon, Oxford, UK), with a sampling frequency of $100 \mathrm{~Hz}$. Furthermore, an accelerometer (Trigno Wireless, Delsys, Natick, MA, USA) was attached at the 7th cervical vertebra to record the acceleration of the trunk sampled at $148 \mathrm{~Hz}$.

The participants performed a tandem walking task on two surfaces: the level ground and a slightly-raised balance beam. On the level ground surface, participants walked longitudinally over a straight line, which had been placed on the ground, and which measured $5 \mathrm{~m}$ in length by $0.02 \mathrm{~m}$ wide. The purpose of this line was to replicate the condition used in Costa et al.'s study [7]. For the balance beam condition, participants walked longitudinally over a narrow wooden board, with the following dimensions: $0.06 \mathrm{~m}$ high, by $0.08 \mathrm{~m}$ wide, by $5 \mathrm{~m}$ long. It is important to note that the width of the balance beam is smaller than the foot width, which reduces the amount of contact surface, and, consequently, the lateral base of support for the foot. All participants walked barefoot to avoid the potential influence of footwear. Although this condition might have caused foot discomfort, none of the participants reported this during or after completing the experiment.

For both surfaces, the participants performed under two conditions: with and without the anchors. In the condition with the anchors, 
A)

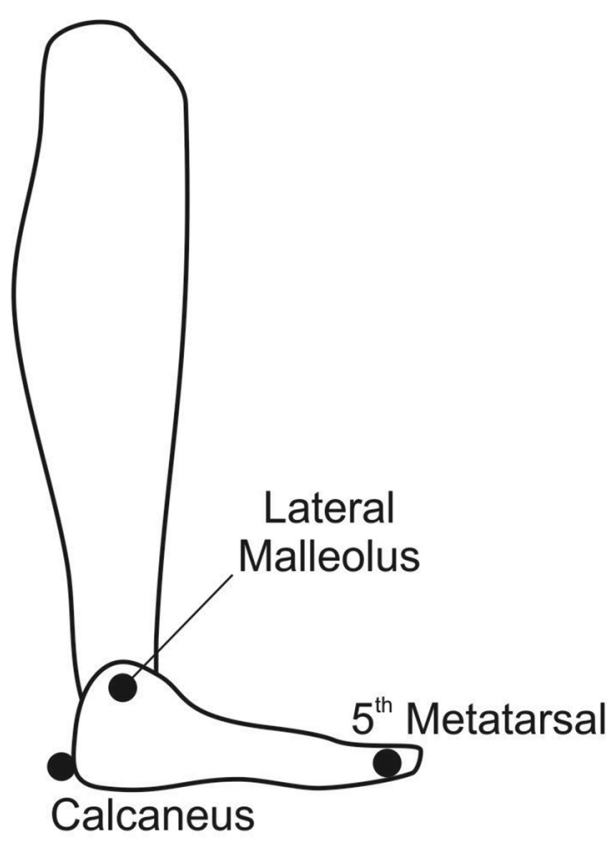

B)

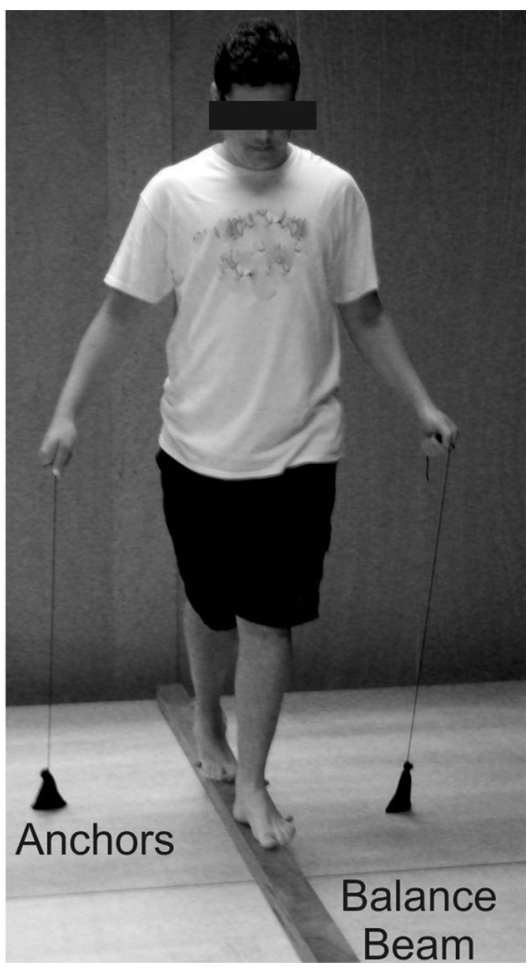

Fig. 1. (A) Illustration showing the location of the markers' placement. (B) Picture showing a young adult tandem-walking on the balance beam while dragging the anchors. participants held, in each hand, a flexible cable with a $125 \mathrm{~g}$ mass (a small cloth bag filled with bird shot) attached to the other end. When using the anchors, participants dragged the $125 \mathrm{~g}$ masses, one in each hand, while keeping them in contact with the ground and the cables taut (Fig. 1B). For walking tasks both with and without anchors, the arms were similarly positioned at the sides of the body. Participants performed five trials for each condition, totaling twenty trials. These trials were all randomized, with a $30 \mathrm{~s}$ interval between trials to prevent fatigue.

\subsection{Data analysis}

The markers' coordinates were filtered by a 4th order, low-pass, digital Butterworth filter, with a cutoff frequency of $6 \mathrm{~Hz}$. These markers were used to calculate the spatiotemporal gait parameters: step speed, and single- and double-support duration. Heel contacts and toeoffs were determined by visual inspection of the stick figure of the foot produced by the three markers [27]. We used the four central steps from the walkers' path to compute the spatiotemporal gait parameters, which corresponds to $\sim 50 \%$ of the total walking path. This option was designed to minimize the effect of the acceleration and deceleration phases. Step speed was computed as the ratio between step length and step duration. Step length corresponded to the difference between the anterior-posterior (AP) coordinates of successive heel contacts. Step duration was calculated as the time difference between successive heel contacts. Single-support duration was calculated as the time difference between the toe-off and the heel contact of the same foot on the surface. Double-support duration was computed as the time difference between the heel contact of the ipsilateral foot and the toe-off of the contralateral foot.

The acceleration data were filtered by a 4th order, low-pass, digital Butterworth filter, with a cutoff frequency of $20 \mathrm{~Hz}$. We used the mathematical algorithm proposed by Moe-Nilssen [28] for transforming the acceleration data into a vertical-horizontal coordinate system. After this transformation, we computed the root mean square (RMS) of the acceleration data in the medial-lateral (ML) direction, or frontal plane, using the four intermediate steps. We calculated RMS for each $1 \mathrm{~s}$ window and, afterwards, the mean value of these windows. The RMS is a measure of the amount of the dispersion into the time-series and its increase is indicative of poor balance control [29].

\subsection{Statistical analysis}

We used the mean value of the five trials for each condition for statistical analysis. In the text and figures, we reported the mean (M) and standard error of the mean (SEM). We performed a 3-way ANOVA ( 2 groups $\times 2$ surfaces $\times 2$ anchor conditions), with repeated measures in the last two factors. We also analyzed the variability between trials. In this case, we computed the standard deviation of the five trials and ran the same statistical model for all dependent variables. We used the least significant difference (LSD) as the post-hoc analysis. We also reported the eta-squared $\left(\eta^{2}\right)$ for the ANOVA. The significance level was .05 .

\section{Results}

Individuals in both groups were physically active, according to the Baecke Questionnaire (Table 1). The older adult group exhibited unimpaired cognitive functions, functional balance, and hand and foot tactile sensitivity. All participants could perform both walking tasks without problems.

\subsection{Spatiotemporal gait variables}

For the single-support duration, ANOVA showed no main or interaction effects involving the anchor conditions. It showed, however, a main effect for surface $\left(F_{1,32}=6.1, p=.019, \eta^{2}=.160\right)$ and an interaction between group and surface $\left(F_{1,32}=5.4, p=.027, \eta^{2}=.143\right)$. As the interaction shows, single support duration increased only for the older adults when walking on the balance beam (Fig. 2A). The variability analysis for the single-support duration revealed an interaction only between group and surface $\left(F_{1,32}=7.0, p=.012, \eta^{2}=.180\right)$. The older adults exhibited higher variability $(\mathrm{M}=0.18 \mathrm{~s}, \mathrm{SEM}=0.04)$ in the balance beam than the young adults $(\mathrm{M}=0.06 \mathrm{~s}, \mathrm{SEM}=0.04)$. The 
Table 1

Mean and standard deviation $( \pm$ ) of the physical, behavioral, and perceptual variables for the participants of both groups.

\begin{tabular}{|c|c|c|}
\hline & Older Adults & Younger Adults \\
\hline $\mathrm{n}$ & 17 & 17 \\
\hline Age (years) & $69.6 \pm 3.6$ & $21.8 \pm 2.7$ \\
\hline Height (m) & $1.63 \pm 0.09$ & $1.73 \pm 0.09$ \\
\hline Body Mass (kg) & $69.9 \pm 14.1$ & $74.3 \pm 13$ \\
\hline Physical Activity Level ${ }^{\mathrm{a}}$ & $7.9 \pm 3.4$ & $8.7 \pm 1.1$ \\
\hline Mini-Mental State Examination ${ }^{\mathrm{b}}$ & $27.5 \pm 2.3$ & --- \\
\hline MiniBESTest $^{c}$ & $26.9 \pm 1.0$ & --- \\
\hline Tactile Sensitivity - Right Hand ${ }^{\mathrm{d}}$ & $2.73 \pm 0.09$ & --- \\
\hline Tactile Sensitivity - Left Hand ${ }^{\mathrm{d}}$ & $2.72 \pm 0.09$ & --- \\
\hline Tactile Sensitivity - Right Foot ${ }^{\mathrm{d}}$ & $3.17 \pm 0.25$ & --- \\
\hline Tactile Sensitivity - Left Foot ${ }^{\mathrm{d}}$ & $3.23 \pm 0.21$ & --- \\
\hline
\end{tabular}

a Scores close to zero indicate a low level of physical activity, as measured by the Baecke Questionnaire for Younger Adults and the Modified Baecke Questionnaire for Older Adults.

b Scores close to 30 points (maximum punctuation) indicate absence of cognitive deficit.

c Scores close to 28 points (maximum punctuation) indicate low risk for falls.

${ }^{\mathrm{d}}$ Values close to 2.7 correspond to sensitivity to the green monofilament $(0.05 \mathrm{~g})$, which is considered a normal sensitivity index for hand and foot. Values close to 3.3 correspond to sensitivity to the blue monofilament $(0.2 \mathrm{~g})$, which are considered a normal sensitivity index for the foot.

older adults also increased variability on the balance beam as compared to the ground $(\mathrm{M}=0.09$, $\mathrm{SEM}=0.03)$.

For the double-support duration, there was no main effect of anchor and no interaction involving anchor and the other factors. However, we found a main effect for surface $\left(F_{1,32}=5.2, p=.030, \eta^{2}=.139\right)$, group $\left(F_{1,32}=4.4, p=.043, \eta^{2}=.122\right)$ and an interaction between group and surface $\left(F_{1,32}=4.7, p=.038, \eta^{2}=.128\right)$. As shown by the interaction, only the older adults increased the double-support duration on the balance beam surface (Fig. 2B). For the variability analysis, the ANOVA identified an interaction effect only between group and surface $\left(F_{1,32}=4.9, p=.034, \eta^{2}=.134\right)$. The older adults increased the variability of the double-support duration when walking on the balance beam $(\mathrm{M}=0.10 \mathrm{~s}, \mathrm{SEM}=0.03)$ as compared to walking on the ground $(\mathrm{M}=0.05 \mathrm{~s}, \mathrm{SEM}=0.02)$.

For step speed, ANOVA identified main effects for group $\left(F_{1,32}=9.3, p=.005, \eta^{2}=.226\right)$, surface $\left(F_{1,32}=60.6, p<.0001\right.$, $\left.\eta^{2}=.654\right)$, anchor $\left(F_{1,32}=18.8, p<.0001, \eta^{2}=.370\right)$, and an interaction between group and surface $\left(F_{1,32}=14.7, p=.001\right.$, $\eta^{2}=.315$ ). As the interaction shows, step speed was greater for the young adults than for the older adults in both surfaces, and it was greater on the level ground surface than on the balance beam, but the older adults reduced their step speed when walking on the balance beam as compared to the young adults (Fig. 2C). In addition, use of the anchors reduced step speed $(\mathrm{M}=0.95 \mathrm{~cm} / \mathrm{s}, \mathrm{SEM}=0.03)$ as compared to the condition without the anchors $(M=0.99 \mathrm{~cm} / \mathrm{s}, \mathrm{SEM}=0.03)$. The ANOVA for the step speed variability identified main effect only for surface $\left(F_{1,32}=5.1, p=.030, \eta^{2}=.138\right)$, showing more variability for the balance beam $(\mathrm{M}=0.10 \mathrm{~cm} / \mathrm{s}, \mathrm{SEM}=0.01)$ than for the ground $(\mathrm{M}=0.09 \mathrm{~cm} / \mathrm{s}, \mathrm{SEM}=0.01)$.

\subsection{Trunk acceleration}

We found only main effects for group $\left(F_{1,32}=8.8, p=.006\right.$, $\left.\eta^{2}=.215\right)$ and anchor $\left(F_{1,32}=4.6, p=.040, \eta^{2}=.125\right)$ for the RMS in the ML direction. RMS was smaller for the young adults than for the older adults (Fig. 2D). Yet, RMS decreased with the use of the anchors as compared to the condition without the use of this tool (Fig. 2E). As there was no interaction between group and anchor $\left(F_{1,32}=0.5\right.$,
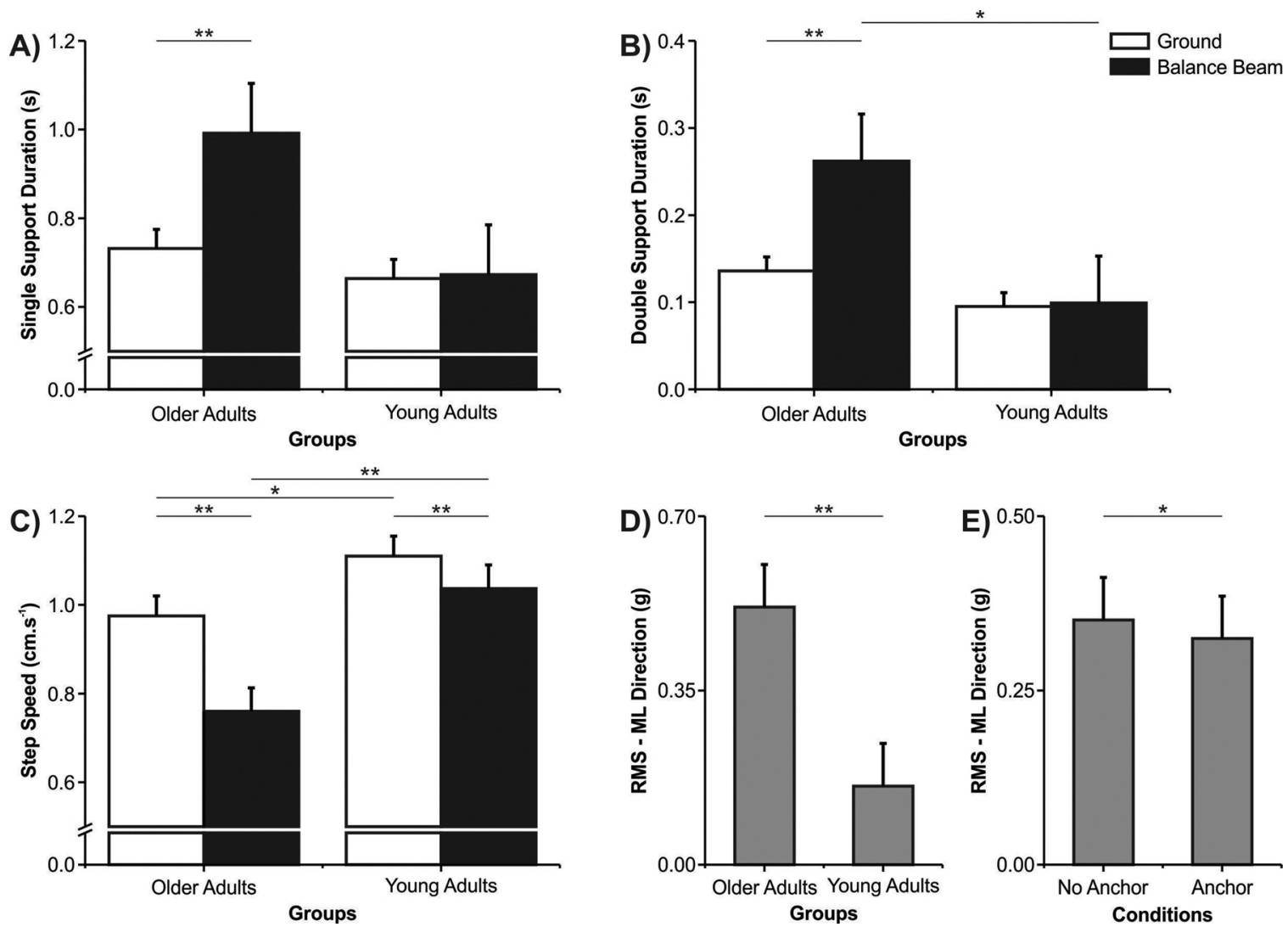

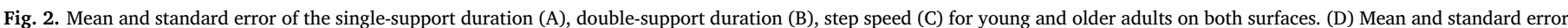

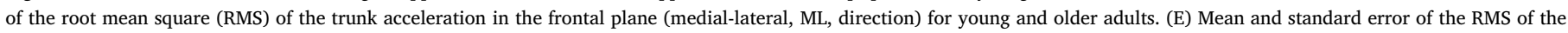
trunk acceleration in the frontal plane (ML direction), without and with the anchors. ${ }^{*} p<.05,{ }^{* *} p<.01$. 
$\left.p=.472, \quad \eta^{2}=.016\right)$ or between group, surface, and anchor $\left(F_{1,32}=1.2, p=.284, \eta^{2}=.036\right)$ the reduction in RMS occurred for both groups. The reduced step speed with the use of the anchors could affect the RMS reduction as well. Therefore, we calculated an ANOVA using the mean step speed with the anchors as a covariate. We identified a main effect for group $\left(F_{1,31}=13.1, p=.001, \eta^{2}=.296\right)$ and a marginal main effect for anchor $\left(F_{1,31}=3.0, p=.093, \eta^{2}=.088\right)$. Thus, the reduction in RMS by anchors is caused in part by the step speed reduction, although the anchors seem to have a direct effect on the RMS as well.

The ANOVA for the variability analysis revealed main effects for group $\left(F_{1,32}=12.5, p=.001, \eta^{2}=.281\right)$ and surface $\left(F_{1,32}=5.0\right.$, $\left.p=.033, \eta^{2}=.135\right)$. The variability between trials was larger for the older adults $(\mathrm{M}=0.15 \mathrm{~g}, \mathrm{SEM}=0.02)$ as compared to the young adults $(\mathrm{M}=0.03 \mathrm{~g}, \mathrm{SEM}=0.02)$. The variability was also greater in the balance beam condition $(\mathrm{M}=0.11 \mathrm{~g}, \mathrm{SEM}=0.02)$ as compared to the ground surface $(\mathrm{M}=0.08 \mathrm{~g}$, $\mathrm{SEM}=0.01)$.

\section{Discussion}

Our purpose was to assess balance control-via analysis of trunk sway in the frontal plane and spatiotemporal gait parameters of walking-in young and older adults, in tandem walking tasks with two levels of difficulty (i.e., ground and balance beam), while using the anchor system. Our results showed that, for the older adults, walking on the balance beam was more difficult than walking on the level ground surface as was reflected in increased single- and double-support duration and reduced step speed. We found that the anchors did not affect the single- and double-support duration in either group, although it reduced step speed. On the other hand, we found that the anchors reduced, at least partially, trunk sway acceleration in the frontal plane regardless of the difficulty level of the walking task. Although the older adults exhibited more difficulty walking on the balance beam than did the young adults, both groups used the anchors to reduce trunk acceleration RMS, although part of this reduction was due to gait speed reduction.

The narrow balance beam surface increased the difficulty of the walking task, especially for the older adults. The step speed change suggests a more conservative control strategy by both groups when they walked on the balance beam. As might be expected, gait speed typically reduces in situations of increasing level of difficulty of the walking task $[1,30]$. When the older adults walked on the balance beam, their greater reduction in step speed and larger increments of single- and double-support duration indicate that they used a more cautious strategy to deal with surface constraints.

In contrast to the spatiotemporal gait parameters, trunk acceleration RMS in the frontal plane showed no difference between the level ground surface and balance beam. The RMS parameter shows that the young individuals likely were not challenged enough by the task constraints of the two surfaces, and, therefore, their trunk oscillation was maintained relatively unaltered. Also, despite the large group differences, the older adults did not show changes in the RMS resulting from the task constraints. It is possible that the task constraints of both surfaces have equal outcomes, but a different meaning in the analysis of the older individuals' performance. Because spatiotemporal parameters were quite different for the older adults, maintaining the trunk acceleration constant in face of the challenging tasks could be interpreted to be a result of stiffness and the freezing of degrees of freedom at the trunk level, although distal joint kinematics also could show a tendency toward stiffness.

Because the young adults were equally affected by both task contexts, their spatiotemporal parameters as well as trunk acceleration show little change, as compared to the older adults. It is possible that, on the balance beam, the older participants adjusted the spatiotemporal parameters of their gait to limit the changes in trunk acceleration in the frontal plane. Additionally, the older adults exhibited higher trunk acceleration sway in the frontal plane than did the young adults, although this result was not influenced by the different tasks. This finding agrees with prior studies that showed an increase in trunk variability with the progression of age [3,4]. In addition, studies have shown that trunk acceleration variability during walking discriminates between frail and fit older adults [5]. This variability is a predictor of falls in older people [2], emphasizing the importance of trunk control to the successful performance of locomotion.

The use of the anchor system reduced step speed. A recent review showed that the effect of haptic input on walking speed is not consistent and that future studies should more fully investigate the interaction between the addition of haptic input and walking speed [31]. It seems plausible that, due to the exploratory nature of haptic anchoring, an individual's reduction in step speed while walking with the anchor tool might be part of a search for a safe and stable gait [1]. Performing a walking task at slower pace intrinsically increases demands for the postural control system to maintain stability as muscle tone of body segments and trunk are likely to increase, with some phases of the gait (i.e., single- and double-support phases) reflecting a more controlled movement, and with the swing phase, perhaps, resulting in a short, ballistic pattern. These aspects are adaptive responses to the task context; therefore, here, a more complex demand is embedded in the haptic anchoring outcome. Furthermore, attentional demands associated with the use of the anchors may have affected the step speed. It might prove useful to compare the effects of walking at different speeds and the influence of the anchor system on step speed and gait stability.

An important result of our study was the reduction of trunk acceleration RMS in the frontal plane with the use of the anchors, which is consistent with our prior findings [7] and of other studies that used either the light touch or the anchor paradigms [12,14]. Despite the reduction of the step speed, the marginal effect of the trunk acceleration RMS with the step speed as a covariate shows a tendency toward trunk sway reduction due to the use of the anchor system. Prior studies have shown that the use of the anchors was beneficial in reducing body sway in young and older adults while they maintained an upright posture $[6,8,9]$. Our results confirm that the benefits observed with the use of the anchors in quiet standing tasks are extended to dynamic tasks, whether simple or more challenging. Perhaps in even more challenging situations, such as requiring participants to walk on a balance beam without vision or on one that is narrower than that used in the present study, the effects of the anchor system could be more easily differentiated. Therefore, future studies might investigate the effects of using the anchor system in these conditions. However, in practical clinical applications, the improvements observed in this study might prove to be useful, since older adults are commonly known to exhibit declines in their balance control.

Interestingly, the effect of using the anchors was similar for both age groups and in both surface conditions. Despite the gradual loss of tactile sensory receptors resulting from the aging process [32], which leads to worsening of performance by these individuals in perceptual discriminative tasks $[33,34,35]$, the benefits that the anchors provided to the haptic input process is comparable to those that were obtained by young adults in an earlier study [36]. Pereira and Moraes [36] found that the use of the anchors reduced body sway similarly in both groups when standing with feet-together. They suggested that the older adults could obtain haptic information, and, like young adults, they can successfully integrate this additional information to reduce body sway.

\section{Conclusion}

Our results suggest that the anchors contributed, at least partially, to reducing the trunk acceleration in the frontal plane, which facilitated the young and older participants' tandem walking performance, regardless of the surface. We observed that task demands via surface restrictions were not sufficient to distinctively modify the locomotion variables for either group. Thus, varying the difficulty of the task had 
no influence on the way in which participants used the anchor system while tandem walking. The integration of haptic information from the anchor system into the postural control mechanism likely requires a task context that presents a sufficient challenge-that is, one that introduces a new behavioral demand-therefore resulting in pressure to form a functional adaptation.

\section{Acknowledgements}

This study was supported by the São Paulo Research Foundation (FAPESP Brazil - Grants 2013/23791-3 and 2015/02404-7). We also would like to thank Debra Frances Campbell, PhD, for her kindness in revising the English manuscript.

\section{References}

[1] M. Schrager, V.E. Kelly, R. Price, L. Ferrucci, A. Shumway-Cook, The effects of age on medio-lateral stability during normal and narrow base walking, Gait Posture 28 (2008) 466-471.

[2] F.B. Mancini, M. Horak, Differentiate balance deficits, Eur. J. Phys. Rehabil. Med. 46 (2010) 239-248.

[3] M. Arvin, M. Mazaheri, J.M. Marco, M. Pijnappels, B.J. Burger, S.M.P. Verschueren, J.H. Van Dieën, Effects of narrow base gait on mediolateral balance control in young and older adults, J. Biomech. 49 (2016) 264-1267.

[4] D.S. Marigold, A.E. Patla, Age-related changes in gait for multi-surface terrain, Gait Posture 27 (2008) 689-696.

[5] R. Moe-Nilssen, J.L. Helbostad, Interstride trunk acceleration variability but not step width variability can differentiate between fit and frail older adults, Gait Posture 21 (2005) 164-170.

[6] E. Mauerberg-deCastro, Developing an anchor system to enhance postural control, Motor Control 8 (2004) 339-358.

[7] A.A.S. Costa, P.A.R. Manciopi, E. Mauerberg-deCastro, R. Moraes, Haptic information provided by the anchor system reduces trunk sway acceleration in the frontal plane during tandem walking in older adults, Neurosci. Lett. 609 (2015) 1-6.

[8] R. Moraes, E. Mauerberg-decastro, O uso de ferramenta não-rígida reduz a oscilação corporal em indivíduos idosos [The use of a non-rigid tool reduces body sway in older individuals], Motriz 15 (2009) 263-272.

[9] M.B.Z. Freitas, E. Mauerberg-Decastro, R. Moraes, Intermittent use of an anchor system improves postural control in healthy older adults, Gait Posture 38 (2013) 433-437.

[10] E. Mauerberg-deCastro, R. Moraes, C. Tavares, G. Figueiredo, S. Pacheco, T. Costa, Haptic anchoring and human postural control, Psychol. Neurosci. 7 (2014) 301-318.

[11] T. Calve, E. Mauerberg-de Castro, Contribuição da percepção háptica no controle postural de crianças, Motriz 11 (2005) 199-204.

[12] I. Hedayat, J. Lanovaz, R. Moraes, A. Oates, Different haptic tools reduce trunk velocity in the frontal plane during walking, but haptic anchors have advantages over lightly touching a railing, Exp. Brain Res. 235 (2017) 1731-1739.

[13] N.D. Boyé, F.U. Mattace-Raso, N. Van der Velde, E.M. Van Lieshout, O.J. De Vries, K.A. Hartholt, A.J. Kerver, M.M. Bruijninckx, T.J. Van der Cammen, P. Patka, E.F. Van, Beeck; IMPROveFALL trial collaborators. Circumstances leading to injurious falls in older men and women in the Netherlands, Injury 45 (2014) $1224-1230$.

[14] K. Bingenheimer, J.J. Temprado, M. Harnagea, N. Bricot, P. Villani, E. Berton, Effects of a light touch on fixed or mobile supports on gait parameters in visually restricted young adults, Neurosci. Lett. 589 (2015) 176-180.
[15] R. Dickstein, Y. Laufer, Light touch and center of mass stability during treadmill locomotion, Gait Posture 20 (2004) 41-47.

[16] E. Kodesh, F. Falash, E. Sprecher, R. Dickstein, Light touch and medio-lateral postural stability during short distance gait, Neurosci. Lett. 584 (2015) 378-381.

[17] E. Mauerberg-deCastro, R. Moraes, D.F. Campbell, Short-term effects of the use of non-rigid tools for postural control by adults with intellectual disabilities, Motor Control 16 (2012) 131-143.

[18] A. Domingo, D.P. Ferris, Effects of physical guidance on short-term learning of walking on a narrow beam, Gait Posture 30 (2009) 464-468.

[19] F.B. Horak, M. Dozza, R. Peterka, L. Chiari, C. Wall, Vibrotactile biofeedback improves tandem gait in patients with unilateral vestibular loss, Ann. N. Y. Acad. Sci. 1164 (2009) 279-281.

[20] A. Sawers, L.H. Ting, Beam walking can detect differences in walking balance proficiency across a range of sensorimotor abilities, Gait Posture 41 (2015) 619-623.

[21] E. Mauerberg-deCastro, C.S. Lucena, B.W. Cuba, R.C. Boni, D.F. Campbell, R. Moraes, Haptic stabilization of posture in adults with intellectual disabilities using a nonrigid tool, APAQ 27 (2010) 208-225.

[22] J.A.H. Baecke, J. Burema, J.E.R. Frijters, A short questionnaire for the measurement of habitual physical activity in epidemiological studies, Am. J. Clin. Nutr. 36 (1982) 936-942.

[23] W.A. Voorrips, L.E. Ravelli, A.C. Dongelmans, P.C.C. Deurenberg, P. Van Staveren, A physical activity questionnaire for the elderly, Med. Sci. Sports Exerc. 23 (1991) 974-979.

[24] S.M.D. Brucki, R. Nitrin, P. Caramelli, P.H.F. Bertolucci, I.H. Okamoto, Sugestões para o uso do mini-exame do estado mental no Brasil [Suggestions for utilization of the mini-mental state examination in Brazil], Arq. Neuropsiquiatr. 61 (2003) 777-781.

[25] A. Franchignoni, F. Horak, F. Godi, M. Nardone, A. Giordano, Using psychometric techniques to improve the balance evaluation systems test: the mini-BESTest, J. Rehabil. Med. 42 (2010) 323-331.

[26] L.F. Lehman, M.B. Orsini, A.R. Nicholl, The development and adaptation of the Semmes-Weinstein monofilaments in Brazil, J. Hand Ther. 6 (1993) 290-297.

[27] R. Moraes, F. Allard, A.E. Patla, Validating determinants for an alternate foot placement selection algorithm during human locomotion in cluttered terrain, J. Neurophysiol. 98 (2007) 1928-1940.

[28] R.A. Moe-Nilssen, New method for evaluating motor control in gait underreal-life environmental conditions. Part 1: the instrument, Clin. Biomech. 13 (1998) $320-327$.

[29] R. Moe-Nilssen, A new method for evaluating motor control in gait under real-life environmental conditions. Part 2: gait analysis, Clin. Biomech. 10 (1998) 328-335.

[30] N. Deshpande, F. Zhang, Trunk, head, and step characteristics during normal and narrow-based walking under deteriorated sensory conditions, J. Mot. Behav. 46 (2) (2014) 125-132.

[31] A.R. Oates, L. Hauck, R. Moraes, K.M. Sibley, The effects of haptic input on biomechanical and neurophysiological parameters of walking: a scoping review, Gait Posture. 58 (2017) 232-239.

[32] J.C. Stevens, Aging and spatial acuity of touch, J. Gerontol. Psychol. Sci. 47 (1992) 35-40.

[33] H.B. Skinner, R.L. Barrack, S.D. Cook, Age-related decline in proprioception, Clin. Orthop. Relat. Res. 184 (1984) 208-211.

[34] F.S. Kaplan, J.E. Nixon, M. Reitz, L. Rindfleish, J. Tucker, Age-related changes in proprioception and sensation of joint position, Acta Orthop. Scand. 56 (1985) 72-74.

[35] D.R. Toledo, J. Barela, Sensory and motor differences between young and older adults: somatosensory contribution to postural control, Rev. Bras. Fisioter. 14 (2010) 267-275.

[36] A.B.R. Pereira, R. Moraes, The use of the anchor system reduces postural sway during upright standing irrespective of plantar flexors muscle fatigue in young and older adults, Motor Control 19 (December) (2017) 1-20, http://dx.doi.org/10. 1123/mc.2017-0035 [Epub ahead of print]. 